\title{
A PHYSICAL MODEL FOR THE $0 \lesssim z \lesssim 8$ REDSHIFT EVOLUTION OF THE GALAXY ULTRAVIOLET LUMINOSITY AND STELLAR MASS FUNCTIONS
}

\author{
Sandro Tacchella ${ }^{1}$, Michele Trenti $^{2}$, and C. Marcella Carollo ${ }^{1}$ \\ ${ }^{1}$ Department of Physics, Institute for Astronomy, ETH Zurich, CH-8093 Zurich, Switzerland; tasandro@phys.ethz.ch \\ ${ }^{2}$ Kavli Institute for Cosmology and Institute of Astronomy, University of Cambridge, Madingley Road, Cambridge, CB3 0HA, UK \\ Received 2012 November 12; accepted 2013 April 11; published 2013 April 29
}

\begin{abstract}
We present a model to understand the redshift evolution of the UV luminosity and stellar mass functions of Lyman break galaxies. Our approach is based on the assumption that the luminosity and stellar mass of a galaxy is related to its dark-matter (DM) halo assembly and gas infall rate. Specifically, galaxies experience a burst of star formation at the halo assembly time, followed by a constant star formation rate, representing a secular star formation activity sustained by steady gas accretion. Star formation from steady gas accretion is the dominant contribution to the galaxy UV luminosity at all redshifts. The model is calibrated by constructing a galaxy luminosity versus halo mass relation at $z=4$ via abundance matching. After this luminosity calibration, the model naturally fits the $z=4$ stellar mass function, and correctly predicts the evolution of both luminosity and stellar mass functions from $z=0$ to $z=8$. While the details of star formation efficiency and feedback are hidden within our calibrated luminosity versus halo mass relation, our study highlights that the primary driver of galaxy evolution across cosmic time is the buildup of DM halos, without the need to invoke a redshift-dependent efficiency in converting gas into stars.
\end{abstract}

Key words: cosmology: theory - galaxies: high-redshift - stars: formation

Online-only material: color figures

\section{INTRODUCTION}

The galaxy luminosity function (LF) and the stellar mass function (MF), along with their redshift evolution, summarize key information on galaxy properties and on their evolution with cosmic time. The rest-frame UV $1500 \AA$ LF in particular can be traced with current technology across the whole redshift range from $z \sim 0$ (e.g., Blanton et al. 2003; Arnouts et al. 2005; Oesch et al. 2010) to $z \sim 10$ (e.g., Reddy \& Steidel 2009; Bradley et al. 2012; Oesch et al. 2012; Ellis et al. 2013), the current frontier of detection of Lyman break galaxy populations. Similarly, the stellar MF can be derived from observations in the rest-frame optical (Arnouts et al. 2007; Stark et al. 2013; González et al. 2011). These data enable a self-consistent comparison of starforming galaxies over the entire span of cosmic history.

A powerful approach to link the properties of galaxies to those of their host dark-matter (DM) halos in a $\Lambda$ cold dark matter cosmology is to use halo occupation distribution models, which give the probability that a halo of mass $M_{h}$ hosts a galaxy (Jing et al. 1998; Peacock \& Smith 2000); these can be generalized into a conditional LF modeling, giving the probability that a halo of mass $M_{h}$ hosts a galaxy with luminosity $L$ (van den Bosch et al. 2003; Cooray 2005). This approach provides an $L\left(M_{h}\right)$ relation between galaxy luminosity and DM halo mass, derived at each redshift through "abundance matching" (e.g., Mo \& Fukugita 1996; Vale \& Ostriker 2004), which generally includes a duty cycle parameter so as to populate with UV luminous galaxies only a fraction of DM halos (Cooray 2005; Lee et al. 2009). It is quite successful in providing a description of the LF, but it does not provide a physical explanation for it.

Our approach aims at identifying the key drivers of the evolution of galaxy properties with the least amount of assumptions. We showed in Trenti et al. (2010) that the LF at $z \gtrsim 5$ is successfully modeled by assuming that UV bright galaxies are present, at any cosmic epoch, only in halos assembled within $\Delta t$ $\left(\Delta t \sim 10^{8} \mathrm{Myr}\right)$. This results in a duty cycle which is physically motivated, defined without free parameters, and dependent on redshift and halo mass. While this model well reproduces the observed rest-frame UV LF evolution at very high redshifts, it cannot be extrapolated down to redshifts $z \lesssim 4$ since at such late epochs DM halos older than a few $10^{8} \mathrm{yr}$ are likely to host UV bright galaxies.

In this Letter, we expand the Trenti et al. (2010) model by making the more realistic assumption that, at any epoch, all massive DM halos host a galaxy with a star formation history (SFH) that is related to the time of halo assembly. Note that the duty cycle inserted by Trenti et al. (2010) is not necessary in our model, which adopts a physical prescription to connect the UV luminosity to a given halo. We anchor our model to the observed LF at $z=4$ and evolve it toward higher $(z \approx 8)$ and lower $(z \approx 0)$ redshifts with a simple physical prescription that enables us to explore the origin of the observed UV LF evolution. Our new model features (1) a burst of star formation at halo assembly time, followed by (2) constant star formation with rate inversely proportional to the halo assembly time (halos at a given mass and different redshift accrete the same gas but over a different timescale). These assumptions, calibrated at $z=4$, are able to reproduce the evolution of the LF, cosmic mass density $\left(\rho_{M_{\star}}\right)$, and specific star formation rate (sSFR) across 13 billion years of cosmic time. This good match between model and observations is achieved with a dominant contribution to the UV luminosity, at all epochs, of a continuous mode of star formation fueled by gas accretion.

This Letter is organized as follows. Section 2 summarizes the observational data sets we aim at modeling. Section 3 describes the model and its calibration. Section 4 presents our results and discusses model uncertainty. Section 5 highlights some concluding remarks. We adopt WMAP5 cosmology: $\Omega_{\Lambda, 0}=$ $0.72, \Omega_{m, 0}=0.28, \Omega_{b, 0}=0.0462, \sigma_{8}=0.817, n_{s}=0.96$, and $h=0.7$ (Komatsu et al. 2009).

\section{OBSERVATIONAL DATA}

Figure 1 and Table 1 summarize the literature compilation of observed UV LFs we use, including the parameters for the bestfit Schechter LF functions $\left(\phi(L)=\phi^{*}\left(L / L^{*}\right)^{\alpha} \exp -\left(L / L^{*}\right)\right)$. 

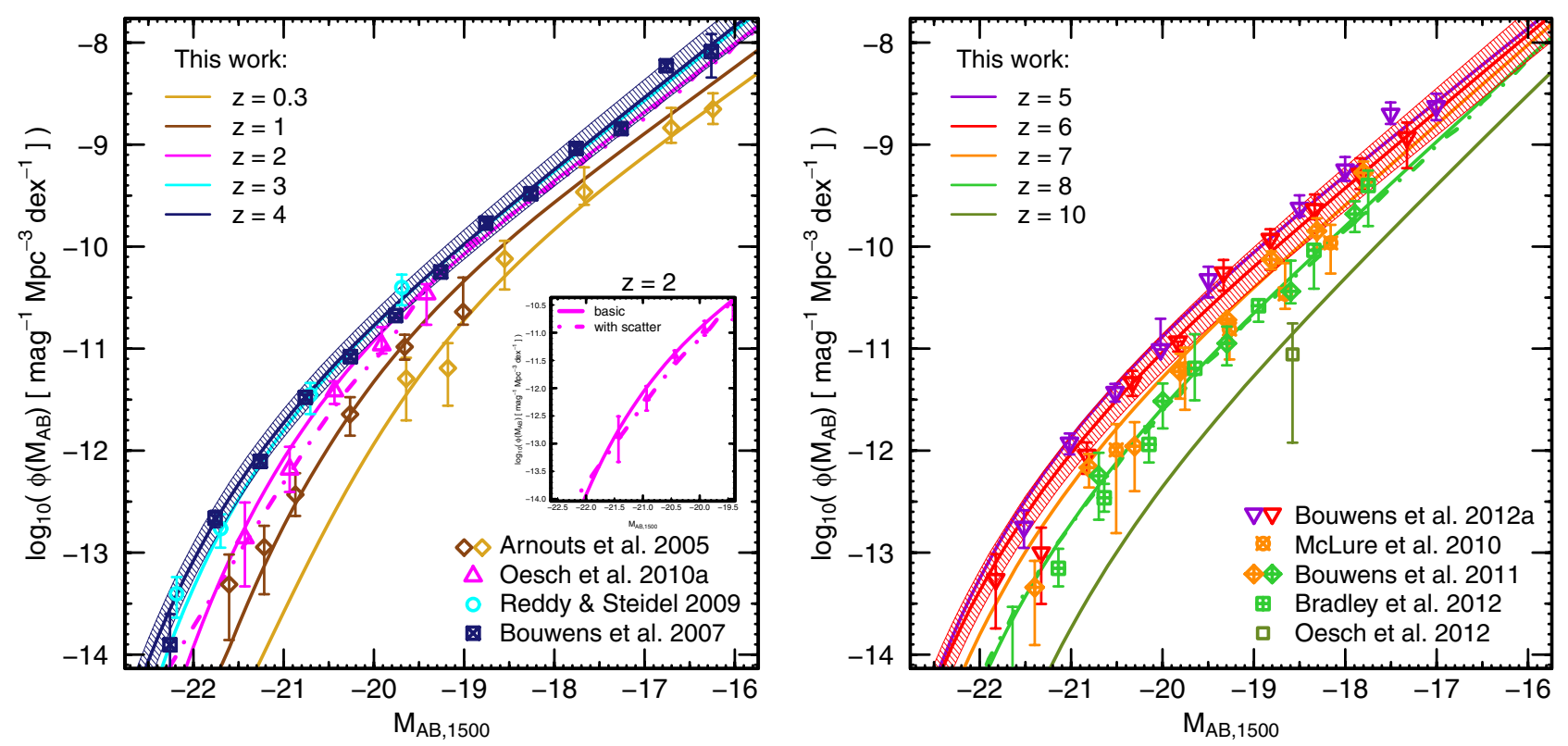

Figure 1. Observed UV luminosity functions (LFs) at low $z$ (left) and high $z$ (right; see Table 1). The shaded areas represent $68 \%$ confidence regions at $z=4$ and $z=6$. The dot-dashed lines show the effects on the LFs at $z=2$ and $z=8$ of accounting for the full probability distribution of the halo assembly time, which induces a scatter in the galaxy luminosity vs. DM halo mass relation (the inset shows enlargement for $z=2$ ); this scatter has a negligible influence only at redshifts $z \gtrsim 4$.

(A color version of this figure is available in the online journal.)

Table 1

Best-fit Schechter Function Parameters for Our Model UV LFs and Observed LFs (from the Literature)

\begin{tabular}{|c|c|c|c|c|c|c|c|c|c|}
\hline \multirow[t]{2}{*}{ Redshift } & \multicolumn{3}{|c|}{ Model Prediction } & \multicolumn{4}{|c|}{ Observed LF } & \multicolumn{2}{|c|}{ UV-continuum Parameters } \\
\hline & $\left(\phi^{*}\right)_{-3}{ }^{\mathrm{a}}$ & $M^{*}$ & $\alpha$ & $\left(\phi^{*}\right)_{-3}{ }^{\mathrm{a}}$ & $M^{*}$ & $\alpha$ & References & $\beta_{M_{\mathrm{UV}}=-19.5}$ & $\left(d \beta / d M_{\mathrm{UV}}\right)$ \\
\hline$z=0.3$ & $4.2 \pm 0.1$ & $-18.9 \pm 0.1$ & $-1.29 \pm 0.05$ & $6.2 \pm 1.8$ & $-18.4 \pm 0.3$ & $-1.19 \pm 0.15$ & (1) & -1.45 & -0.13 \\
\hline$z=1$ & $1.6_{-0.1}^{+0.2}$ & $-19.9 \pm 0.1$ & $-1.63_{-0.02}^{+0.04}$ & $1.1 \pm 0.8$ & $-20.1 \pm 0.5$ & $-1.63 \pm 0.45$ & (1) & -1.55 & -0.13 \\
\hline$z=2$ & $2.2_{-0.1}^{+0.2}$ & $-20.3_{-0.1}^{+0.2}$ & $-1.60_{-0.06}^{+0.04}$ & $2.2 \pm 1.8$ & $-20.2 \pm 0.5$ & $-1.60 \pm 0.51$ & (2) & -1.70 & -0.13 \\
\hline$z=3$ & $1.72 \pm 0.01$ & $-20.9_{-0.1}^{+0.3}$ & $-1.68_{-0.07}^{+0.05}$ & $1.7 \pm 0.5$ & $-21.0 \pm 0.1$ & $-1.73 \pm 0.13$ & (3) & -1.85 & -0.13 \\
\hline$z=4$ & $1.30 \pm 0.01$ & $-21.0_{-0.3}^{+0.2}$ & $-1.73_{-0.05}^{+0.07}$ & $1.3 \pm 0.2$ & $-21.0 \pm 0.1$ & $-1.73 \pm 0.05$ & (4) & -2.00 & -0.13 \\
\hline$z=5$ & $1.4 \pm 0.1$ & $-20.6_{-0.3}^{+0.2}$ & $-1.77_{-0.05}^{+0.11}$ & $1.4_{-0.5}^{+0.7}$ & $-20.6 \pm 0.2$ & $-1.79 \pm 0.12$ & (5) & -2.08 & -0.16 \\
\hline$z=6$ & $1.4 \pm 0.1$ & $-20.4_{-0.2}^{+0.4}$ & $-1.76_{-0.12}^{+0.14}$ & $1.4_{-0.6}^{+1.1}$ & $-20.4 \pm 0.3$ & $-1.73 \pm 0.20$ & (5) & -2.20 & -0.17 \\
\hline$z=7$ & $0.9 \pm 0.1$ & $-20.2 \pm 0.2$ & $-1.84_{-0.17}^{+0.12}$ & $0.9_{-0.4}^{+0.0}$ & $-20.1 \pm 0.3$ & $-2.01 \pm 0.21$ & (6) & -2.27 & -0.21 \\
\hline$z=8$ & $0.5 \pm 0.1$ & $-20.2_{-0.2}^{+0.4}$ & $-1.92_{-0.15}^{+0.11}$ & $0.4_{-0.2}^{+0.4}$ & $-20.3_{-0.3}^{+0.3}$ & $-1.98_{-0.2}^{+0.2}$ & (7) & -2.34 & -0.25 \\
\hline$z=10$ & $0.2 \pm 0.1$ & $-19.74_{-0.5}^{+0.3}$ & $-2.18_{-0.02}^{+0.25}$ & $0.1 \pm 0.1$ & $-19.6^{\mathrm{b}}$ & $-1.73^{\mathrm{b}}$ & (8) & $\ldots c$ & $\ldots c$ \\
\hline
\end{tabular}

Notes. The best-fit Schechter function parameters, as a function of redshift (Column 1), for our model-predicted LFs (Columns 2-4; see Figure 1), and for observed UV LFs (taken from the literature; Columns 5-8). Quoted errors for our model predictions are derived by propagating the uncertainty in the $L\left(M_{h}, z=4\right)$ calibration (see also Figure 3). The last two columns show the adopted slopes $\beta_{M_{\mathrm{UV}}=-19.5}$ and intercepts $d \beta / d M_{\mathrm{UV}}$ to the UV-continuum slope $\beta$ to UV luminosity relationship as in Bouwens et al. (2012a, Table 5).

${ }^{a}$ Units: $10^{-3} \mathrm{Mpc}^{-3}$.

${ }^{\mathrm{b}}$ Values were fixed for the fit of the Schechter function.

${ }^{\mathrm{c}}$ No dust correction at $z \sim 10$.

References. (1) Arnouts et al. 2005; (2) Oesch et al. 2010; (3) Reddy \& Steidel 2009; (4) Bouwens et al. 2007; (5) Bouwens et al. 2012b; (6) Bouwens et al. 2011; (7) Bradley et al. 2012; (8) Oesch et al. 2012.

Observed stellar mass densities $\left(\rho_{M_{*}}\right)$ integrated above $M_{*, \min }=$ $10^{8} M_{\odot}$ and sSFR at $M_{\star}=5 \times 10^{9} M_{\odot}$ are shown in Figure 2. The figure also includes the cosmic SFR density $\dot{\rho}_{M_{\star}}$, obtained by converting, using the Madau et al. (1998) relation, the luminosity density $\rho_{L}$ integrated to $L_{\min }=0.05 L_{z=3}^{*}$ (corresponding to $M_{\mathrm{AB}}=-17.7$ ). Overplotted to observations are our model predictions, obtained with the prescriptions described below.

\section{MODEL DESCRIPTION}

Our model links the UV LF to abundance of DM halos at the same epoch, from $z=0$ to $z \sim 10$, adopting a physical recipe for star formation with dependence on halo assembly time.

\subsection{Halo Assembly Time}

We adopt the halo assembly time (redshift) as defined by Lacey \& Cole (1993) as typical timescale for galaxy formation. The assembly redshift $z_{a}$ of a halo of mass $M_{h}$ at redshift $z$ is the redshift at which the mass of the main progenitor is $M_{h} / 2$, which can be calculated within the extended Press-Schechter formalism (Bond et al. 1991). For this, we use the ellipsoidal collapse model (Sheth et al. 2001), which reproduces well numerical simulation results (Giocoli et al. 2007). We adopt as the fiducial assembly time for each halo the median of the probability distribution associated with each halo (shown in the top-left panel of Figure 3 for different redshifts), but we also 


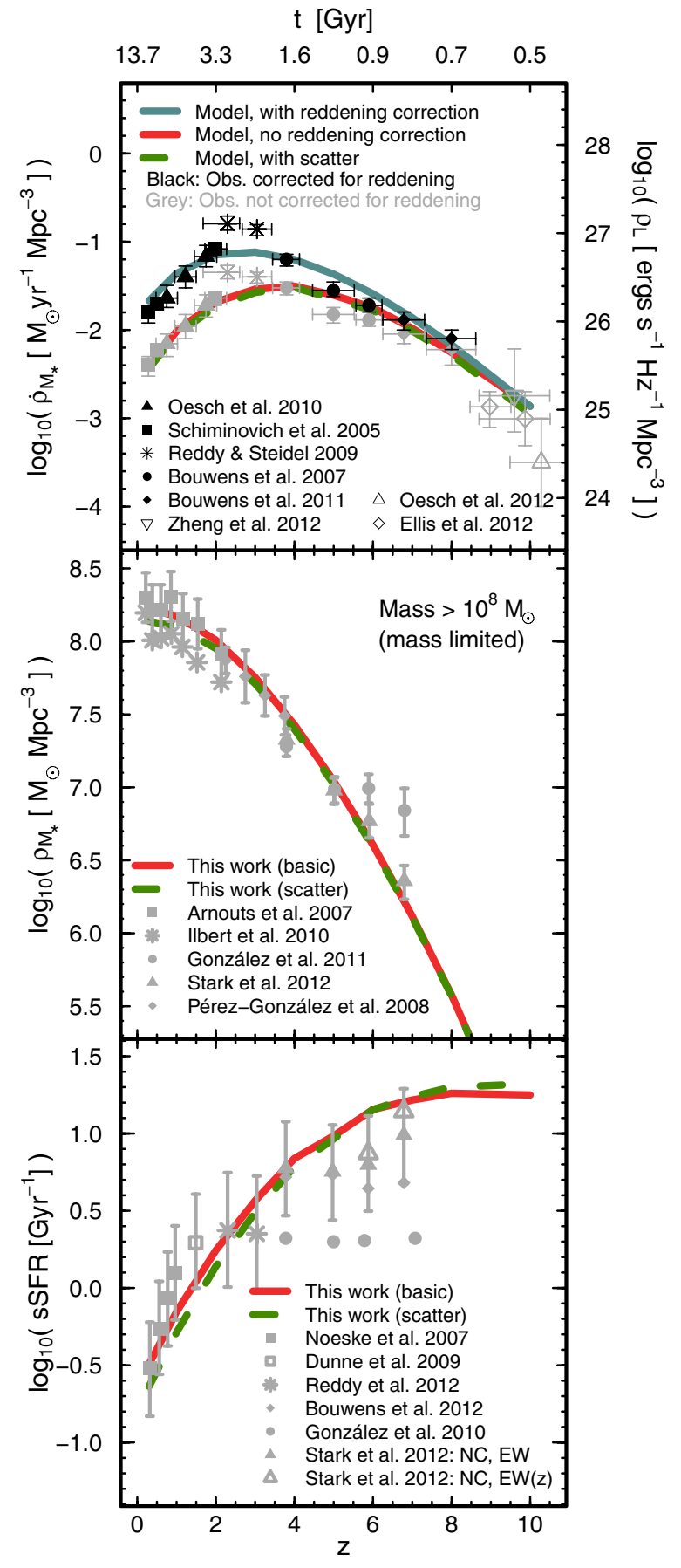

Figure 2. Top panel: star formation rate density $\left(\dot{\rho}_{M_{\star}}\right)$ and luminosity density $\left(\rho_{L}\right)$ derived by integrating the model UV LFs in comparison with the observations before (gray points) and after dust correction (black points). Both $\rho_{L}$ and $\dot{\rho}_{M_{\star}}$ are given for $L \geqslant 0.05 L_{z=3}^{*}\left(M_{\mathrm{AB}} \leqslant-17.7\right)$. Middle: evolution in the stellar mass density $\left(\rho_{M_{\star}}\right)$, computed by integrating the stellar mass function to a fixed stellar mass limit of $10^{8} M_{\odot}$. Bottom: evolution in the specific star formation rate (sSFR) as a function of redshift for a galaxy with $M_{*}=5 \times 10^{9} M_{\odot}$. The red lines show our standard model predictions, while the dashed green lines show the model that includes the full probability distribution of the halo assembly time (i.e., with scatter in the $L\left(M_{h}, z\right)$ relation).

(A color version of this figure is available in the online journal.)

account for the full probability distribution of $z_{a}$ to compute the scatter in the $L\left(M_{h}, z\right)$ relation and validate our simpler assumption of a median value for $z_{a}$. At a given mass, halos are assembled faster at higher $z$, with important consequences on the UV properties of stellar populations.

\subsection{Star Formation Modeling}

We populate halos with stars based on the simple stellar population (SSP) models of Bruzual \& Charlot (2003), adopting a Salpeter initial mass function (Salpeter 1955) between $M_{L}=$ $0.1 M_{\odot}$ and $M_{U}=100 M_{\odot}$. We use constant stellar metallicity $Z=0.02 Z_{\odot}$, neglecting redshift evolution as there is little dependence of the UV luminosity on metallicity. We define as $l(t)$ the resulting luminosity at $1500 \AA$ for an SSP of age $t$ and stellar mass $1 M_{\odot}$.

For a halo at a given redshift, we set the start of the SFH to coincide with the halo assembly time $t_{H}\left(z_{a}\right)$. Specifically, we parameterize the SFHs through a short-duration burst at the halo assembly time, followed by a constant SFR period. This latter term is normalized by $1 / t_{\text {age }}$, with $t_{\text {age }}=t_{H}(z)-t_{H}\left(z_{a}\right)$ and $t_{H}(z)$ the age of the universe at redshift $z$. The "burst mode" integrates the total stellar mass produced from the earliest epochs down to $t_{H}\left(z_{a}\right)$. This integrated contribution of early star formation activity makes up for about half of the stellar mass; as $t_{H}\left(z_{a}\right) \gtrsim 10^{8} \mathrm{yr}$, the burst adds up however relatively little, i.e., $\lesssim 20 \%$, to the UV LF (see Section 4). During the continuous "accretion mode," halos accrete of the order of $M_{h} / 2$ within the $t_{\text {age }}$ timescale. Since $t_{\text {age }}$ depends strongly on $z$ (see Figure 3, top left panel), the accretion rate changes as well: halos of the same mass at higher redshifts have naturally higher accretion rates, as also indicated by other studies (Genel et al. 2008; Dekel et al. 2009). The resulting halo luminosity is

$$
\begin{aligned}
L\left(M_{h}, z\right)= & x \cdot\left[\eta\left(M_{h}\right) M_{h} l\left(t_{\text {age }}\right)\right] \\
& +(1-x) \cdot\left[\varepsilon\left(M_{h}\right) M_{h} \frac{1}{t_{\text {age }}} \int_{0}^{t_{\text {age }}} l(t) d t\right] .
\end{aligned}
$$

The first term $\eta\left(M_{h}\right)$ describes the efficiency of the burst episode, while the second term $\varepsilon\left(M_{h}\right)$ describes the efficiency of the accretion mode. Both efficiencies are assumed to be redshift independent (see Behroozi et al. 2013). The free parameter $x$ controls the relative contribution of the initial burst to the total luminosity of the galaxy at $z=4$.

From Equation (1), the resulting stellar mass, i.e., the time integral of the SFR is

$$
M_{\star}\left(M_{h}\right)=x \cdot \eta\left(M_{h}\right) M_{h}+(1-x) \cdot \varepsilon\left(M_{h}\right) M_{h},
$$

which is used to obtain stellar densities and sSFRs.

\subsection{Dust Extinction}

Dust extinction significantly affects the observed UV flux, especially at $z \lesssim 4$ (see Figure 2 , top panel). Following Smit et al. (2012), for a spectrum modeled as $f_{\lambda} \sim \lambda^{\beta}$, we assume a linear relation between the UV-continuum slope $\beta$ and luminosity $\left(\langle\beta\rangle=\left(d \beta / d M_{\mathrm{UV}}\right)\left(M_{\mathrm{UV}, \mathrm{AB}}+19.5\right)+\beta_{M_{\mathrm{UV}, \mathrm{AB}}}\right)$. Assuming a dependence of UV extinction on $\beta$ as $A_{\mathrm{UV}}=$ $4.43+1.99 \beta$ (Meurer et al. 1999), and a Gaussian distribution for $\beta$ at each $M_{\mathrm{UV}}$ value (with dispersion $\sigma_{\beta}=0.34$ ), the average $\left\langle A_{\mathrm{UV}}\right\rangle$ is given by $\left\langle A_{M_{\mathrm{UV}}}\right\rangle=4.43+0.79 \ln (10) \sigma_{\beta}^{2}+1.99\langle\beta\rangle$. We adopted the value of 0 for any negative $\left\langle A_{\mathrm{UV}}\right\rangle$. Values for $d \beta / d M_{\mathrm{UV}}$ and $\beta_{M_{\mathrm{UV}, \mathrm{AB}}}$ are taken from Table 5 of Bouwens et al. (2012a) and are listed in Table 1. We extrapolated $\beta_{M_{\mathrm{UV}, \mathrm{AB}}}$ to higher and lower redshifts, while letting $d \beta / d M_{\mathrm{UV}}$ constant at the $z=4$ value, since uncertainties in this latter parameter are large. 

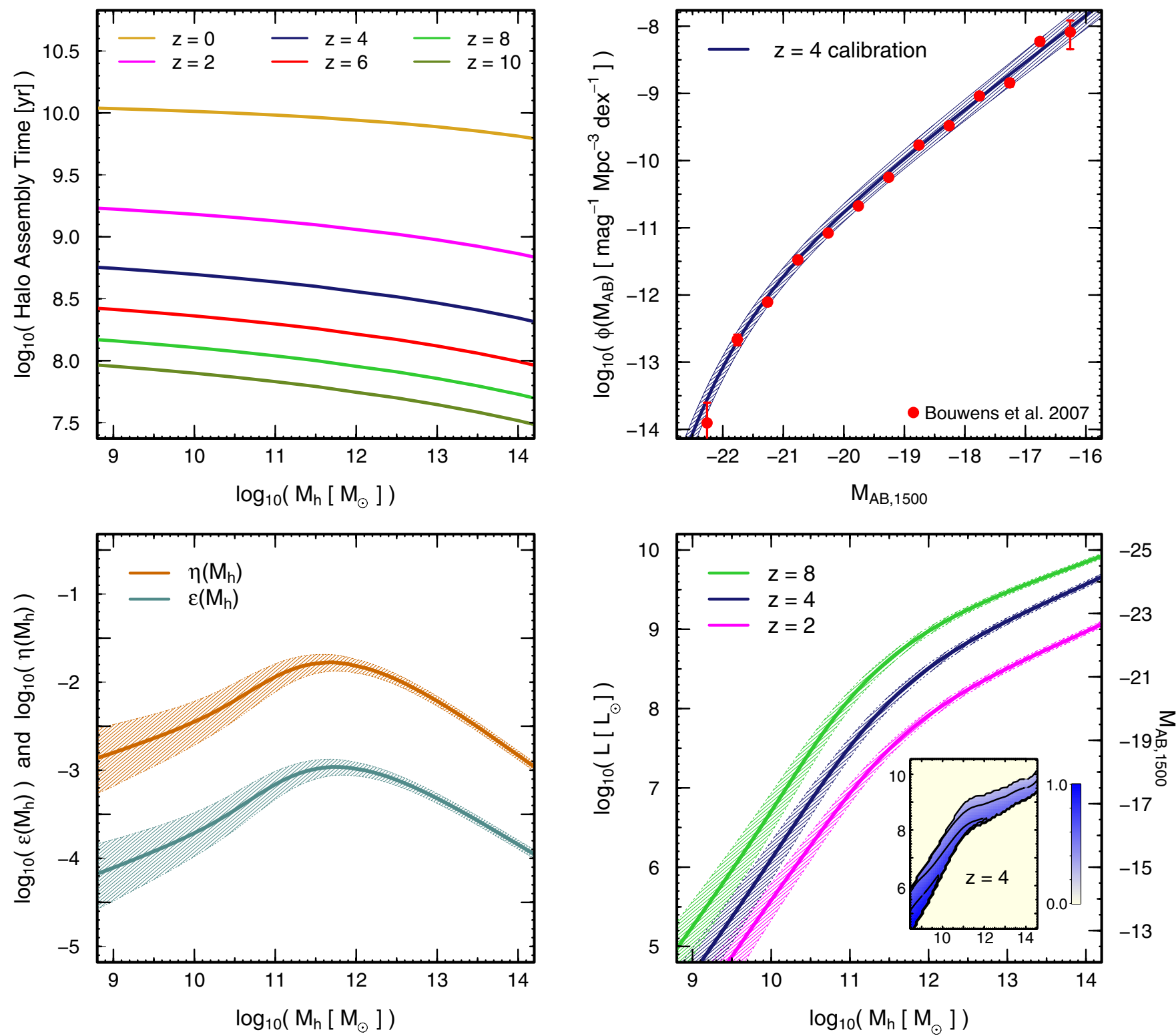

Figure 3. Top left panel: DM halo assembly time $t_{\text {age }}$ vs. halo mass and redshift. Top right panel: calibration of our model using the Bouwens et al. (2007) LF at $z=4$. Bottom left panel: star formation efficiencies $\eta\left(M_{h}\right)$ and $\varepsilon\left(M_{h}\right)$ for the burst and accretion mode, respectively. The highest efficiencies are in the range $M_{h} \sim 10^{11}-10^{12} \mathrm{M}_{\odot}$. Bottom right panel: the relation between galaxy luminosity and DM halo mass, $L\left(M_{h}, z\right)$, plotted at $z=2,4$, and 8 . The inset shows the scatter in the $L\left(M_{h}, z\right)$ relation at $z=4$ produced by sampling the whole probability distribution of halo assembly times. In all panels, shaded areas represent $68 \%$ confidence regions.

(A color version of this figure is available in the online journal.)

\subsection{Model Calibration}

To calibrate $\eta\left(M_{h}\right)$ and $\varepsilon\left(M_{h}\right)$ we perform abundance matching at $z=4$, assuming one galaxy per halo and equating the number of galaxies with luminosity greater than $L$ (after dust correction) to the number of halos with mass greater than $M_{h}$ :

$$
\int_{M_{h}}^{+\infty} n\left(\tilde{M}_{h}, z=4\right) d \tilde{M}_{h}=\int_{L}^{+\infty} \phi(\tilde{L}, z=4) d \tilde{L}
$$

where $n\left(M_{h}, z\right)$ is the MF of DM halos obtained adopting the Sheth \& Tormen (1999) MF. This gives us a luminosity versus halo mass relation at $z=4, L\left(M_{h}, z=4\right)$, shown in the bottom right panel of Figure 3. From this, we can then infer $\eta\left(M_{h}\right)$ and $\varepsilon\left(M_{h}\right)$ by solving Equation (1) (bottom left panel of Figure 3). We calibrate these two quantities independently, so that their linear combination also satisfies $L\left(M_{h}, z=4\right)$ by construction. The shaded areas in both panels represent the uncertainty in the model calibration derived by varying the $z=4 \mathrm{LF}$ parameters within the $1 \sigma$ confidence regions in Figure 3 of Bouwens et al. (2007). From the bottom left panel of Figure 3 it is immediate to see that halos with $M_{h} \sim 10^{11}-10^{12} M_{\odot}$ have the highest specific star formation efficiencies. This is not surprising, given the shapes of the LFs and DM MF.

Our final calibration step is selecting a value for the only free parameter in the model, $x$, i.e., the contribution of the burst to the total luminosity at $z=4$. For this we compute model predictions over the redshift range $0 \lesssim z \lesssim 8$ with varying $x$ values, and adopt the value of $x$ which minimizes the residuals relative to the observed LFs. The best match to observations is given by $x=0.1$, which is a $10 \%$ of contribution from the 

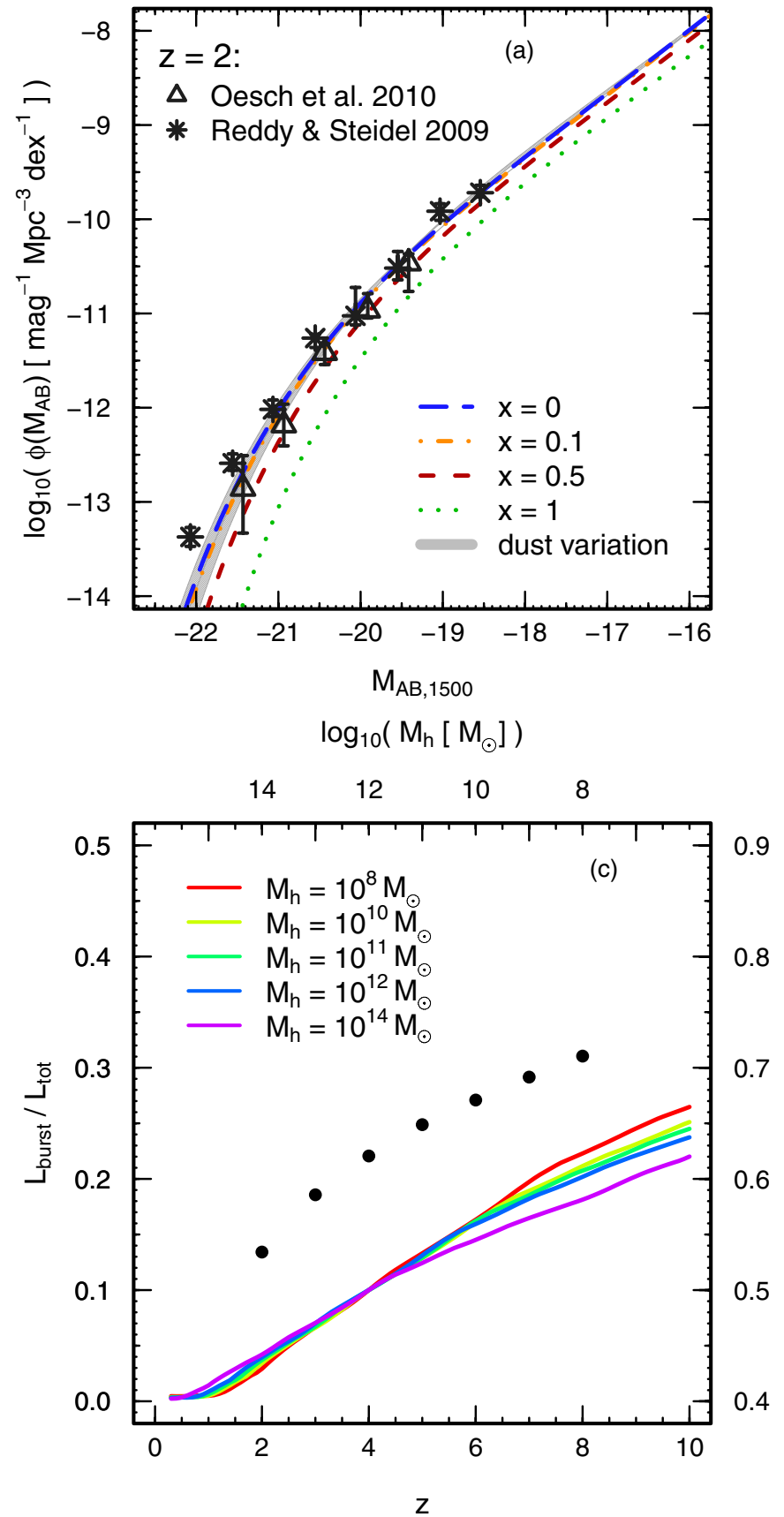
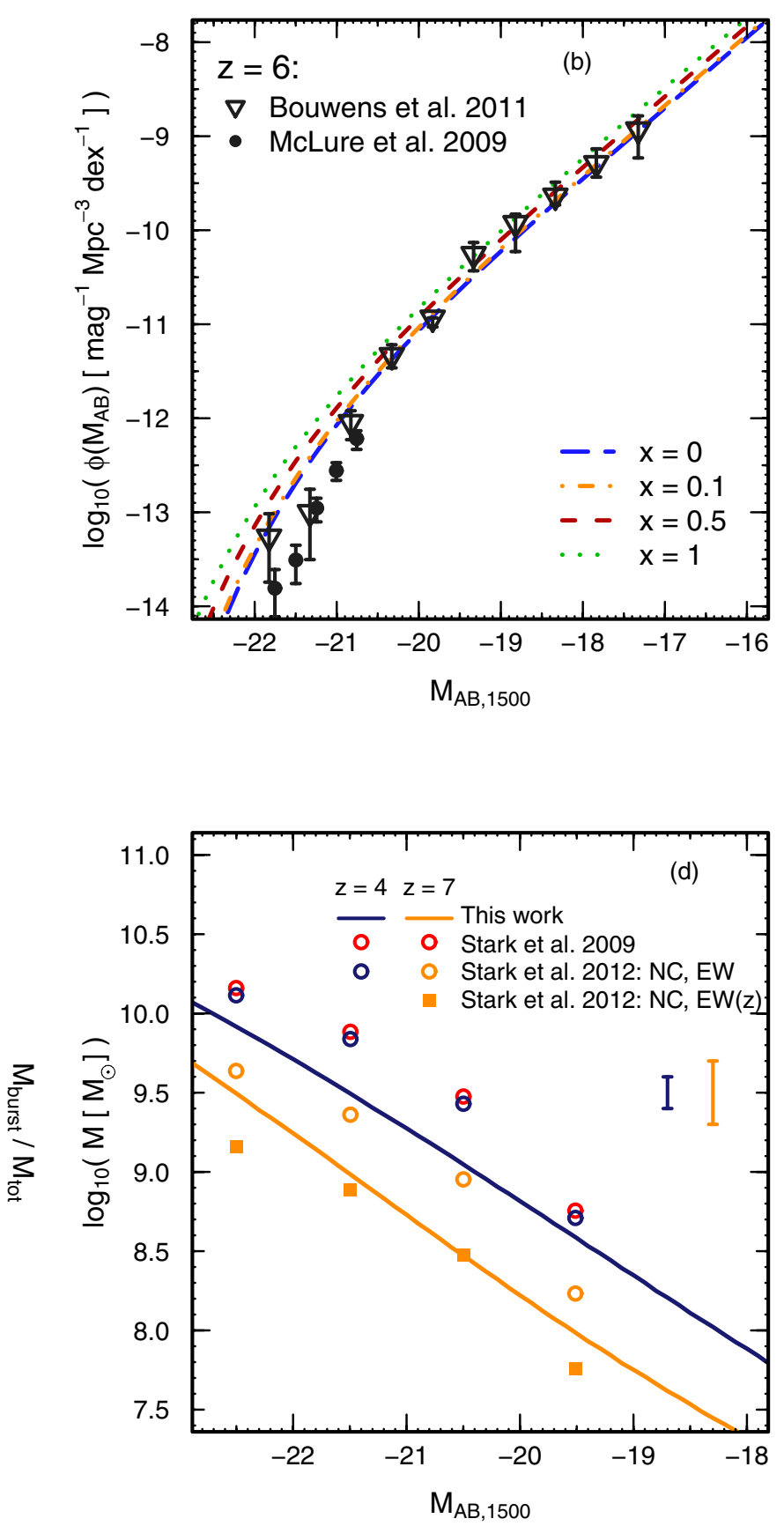

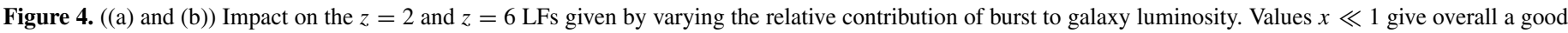

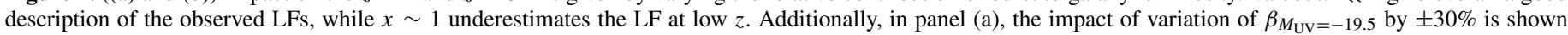

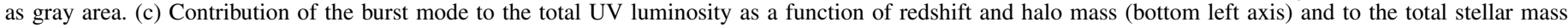

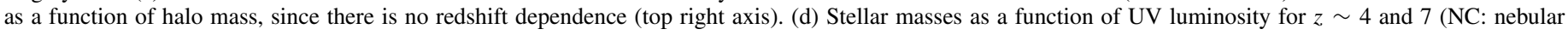
contamination corrected data with equivalent width evolution $(\mathrm{EW}(z))$ and without $(\mathrm{EW})$, respectively).

(A color version of this figure is available in the online journal.)

initial burst to the total halo luminosity at $z=4$; the model is, however, not very sensitive to the exact value for as long as $x \ll 1$ (see the top panels of Figure 4).

\section{RESULTS AND DISCUSSION}

The curves in the bottom right panel of Figure 3 show our predictions for the observed $L\left(M_{h}, z\right)$ relation at different redshifts. A decreasing contribution from dust is the main cause for the brightening of the relation toward higher redshifts at fixed halo mass. Note that these assume a single assembly time for a given halo (see Section 3.1). Taking into account the whole probability distribution for the halo assembly time leads to scatter in the $L\left(M_{h}, z\right)$ relation (shown in the inset of Figure 3, bottom right panel), but such a model has overall similar predictions in terms of the observed LF as shown in Figure 1 for $z=2$ and $z=8$. Therefore, we focus primarily on our canonical model without scatter.

The predictions for the LFs over the $z \lesssim 10$ time span are shown overplotted to the observations in Figure 1. In addition, the model reproduces both low- $z$ and high- $z$ UV LFs remarkably well, suggesting that the evolution of the UV LF across most 
of cosmic time can indeed result from the redshift evolution of the halo MF, coupled with simple SFHs beginning at the halo assembly time.

The model, calibrated to the observed Schechter LF at $z=4$, produces Schechter functions at all other epochs. Furthermore, the predicted LFs well approximate the Schechter functions with the observed best-fit parameters reported in Table 1 (Figure 1). In particular, the model correctly describes the evolution of the faint-end slope $\alpha$, from its shallow low- $z$ value $\alpha(z \approx 0) \sim-1.3$ to the steepening observed at $z \gtrsim 6$, where $\alpha \lesssim-1.7$. At high $z$, the model LFs are similar to the LFs predicted by Trenti et al. (2010); in addition, our new model is also successful in reproducing the observed LFs also at low redshifts, all the way down to $z \simeq 0$, resolving the puzzling quick rise of $\alpha$ from $z \simeq 0$ to 1 (e.g., Oesch et al. 2010).

Figure 2 shows the model predictions for the redshift evolution of the SFR density $\dot{\rho}_{M_{\star}}(z)$ and luminosity density $\rho_{L}(z)$ (top panel). The model-observation agreement for $\dot{\rho}_{M_{\star}}(z)$ is again very good at all epochs from $z \approx 0$ to $z \approx 8$. At $z \sim 10$ the model appears to overpredict $\dot{\rho}_{M_{\star}}$, as measured by Oesch et al. (2012), by $\sim 0.8$ dex. This might be due to very short assembly times for $z \sim 10$ halos ( $t_{\text {age }} \lesssim 10^{8} \mathrm{Myr}$ ), hence to a dominant contribution to the UV light from the very young stellar populations produced in the burst mode. Another possibility is sample variance in the observations. In fact, Zheng et al. (2012) derive from a gravitationally lensed source in CLASH $\dot{\rho}_{M_{\star}}(z=10)=\left(1.8_{-1.1}^{+4.3}\right) \times 10^{-3} M_{\odot} \mathrm{Mpc}^{-3} \mathrm{yr}^{-1}$, in agreement with our model predictions.

Figures 4(a) and (b) show model-observation comparisons for the $z=2$ and $6 \mathrm{LFs}$; the model results are plotted for different values of the burst fractional contribution $x$ to the total UV luminosity, and for different dust extinction corrections (as parameterized by $\beta$ ). Varying $\beta$ within the uncertainty given by Bouwens et al. (2012a) has little impact on the predicted LFs, which are thus robust against uncertainties in the amount and treatment of dust obscuration. In contrast, the predicted LFs do depend on the choice of $x$. As the SFHs are modeled to approach a single episode of star formation at the halo assembly time, i.e., $x \rightarrow 1$, the $L\left(M_{h}, z\right)$ relation shows an increasingly stronger dependence on the assembly time: at low redshift, halos become too faint relative to the observations, and the LFs and $\rho_{L}$ are underestimated; at high redshifts, halos are too UV bright and LFs and $\rho_{L}$ are overestimated.

By construction, the initial burst phase contributes modestly to the UV luminosity of galaxies at all epochs, especially at lower redshifts, i.e., as $t_{\text {age }}$ increases. This is shown in Figure 4(c), which plots the contribution of the burst mode to the total galaxy luminosity for halos of different masses. The redshift evolution of the $L_{\text {burst }} / L_{\text {tot }}$ ratio is faster for smaller halos, as for these $t_{\text {age }}$ evolves faster (Figure 3 ). In contrast, the initial star formation burst, occurring at the time when the halo has already assembled half of its total mass, consistently contributes of the order of a half of the stellar mass budget (Figure 4(c)). Specifically, the contribution of the burst phase to the stellar mass is roughly $50 \%$ at $M_{h} \sim 10^{14} M_{\odot}$, and increases to about $70 \%$ at $M_{h} \sim 10^{9} M_{\odot}$.

Figure 4(d) shows stellar mass as a function of magnitude. The red points show the relation at $z=4$ of Stark et al. (2009), and the blue points are re-normalized for accounting for emission lines (see Stark et al. 2013; de Barros et al. 2012). The model data are slightly below the $z=4$ observations; in contrast, at $z=7$ the model predicts a $M_{\star}-M_{\mathrm{AB}, 1500}$ relation which well matches the data of Stark et al. (2013), once these are corrected for emission line contamination and for a redshift-dependent equivalent width of nebular emission (increasing with increasing redshift).

The middle panel of Figure 2 shows the comparison between model and observations for $\rho_{M_{\star}}$. Overall, the model (with or without scatter in $L\left(M_{h}, z\right)$ ) fits the data well at all redshifts. At $z=7$, we underestimate by $0.25 \mathrm{dex}$ the (not emissioncorrected) González et al. (2011) data point. On the other hand, we are broadly consistent with the Stark et al. (2013) measurements. The sSFR is shown in the bottom panel of Figure 2 and shows overall a good agreement with the observations.

\section{CONCLUSION}

We have presented a model for the evolution of the UV LF based on the simple assumption that all massive DM halos host a galaxy with a SFH that is closely related to the halo assembly time. We specifically adopt for the SFHs the combination of an initial star formation burst at the halo assembly time, representing the integrated galaxy SFHs down to this epoch, plus a constant SFR phase, a proxy for secular, low-level star formation activity fueled by steady gas accretion. While the assumption that each DM halo hosts only one galaxy is clearly a simplification, especially toward lower redshifts, the model is remarkably successful in reproducing major features of the evolving star-forming galaxy population since $z \simeq 8$; this is also due to the fact that massive galaxies which, at later times, will share a common halo will be mostly quenched of their star formation activity. It is remarkable that this simple parameterization reproduces very well the evolution of the UV LFs over the whole cosmic time since $z \simeq 8$ down to $z \simeq 0$, as well as the evolution of the cosmic sSFR, luminosity density, and stellar mass density. In our model, the cosmic SFR density rises and then falls toward lower $z$ naturally as it is observed, which can be explained by the drop in the accretion rate at low redshifts of the individual DM halos-overtaking the increase in abundance of galaxies at $z \lesssim 2$. This demonstrates the key role played by DM halo assembly in shaping the properties of the luminous galaxies.

\section{REFERENCES}

Arnouts, S., Schiminovich, D., Ilbert, O., et al. 2005, ApJ, 619, 43 Arnouts, S., Walcher, C. J., Le Fèvre, O., et al. 2007, A\&A, 476, 137 Behroozi, P. S., Wechsler, R. H., \& Conroy, C. 2013, ApJ, 762, 31 Blanton, M. R., Hogg, D. W., Bahcall, N. A., et al. 2003, ApJ, 592, 819 Bond, J. R., Cole, S., Efstathiou, G., \& Kaiser, N. 1991, ApJ, 379, 440 Bouwens, R. J., Illingworth, G. D., Franx, M., \& Ford, H. 2007, ApJ, 670, 928 Bouwens, R. J., Illingworth, G. D., Oesch, P. A., et al. 2011, ApJ, 737, 90 Bouwens, R. J., Illingworth, G. D., Oesch, P. A., et al. 2012a, ApJ, 754, 83 Bouwens, R. J., Illingworth, G. D., Oesch, P. A., et al. 2012b, ApJ, 752, 5 Bradley, L. D., Trenti, M., Oesch, P. A., et al. 2012, ApJ, 760, 108 Bruzual, G., \& Charlot, S. 2003, MNRAS, 344, 1000 Cooray, A. 2005, MNRAS, 364, 303

de Barros, S., Schaerer, D., \& Stark, D. P. 2012, arXiv:1207.3663 Dekel, A., Birnboim, Y., Engel, G., et al. 2009, Natur, 457, 451 Ellis, R. S., McLure, R. J., Dunlop, J. S., et al. 2013, ApJL, 763, L7 Genel, S., Genzel, R., Bouché, N., et al. 2008, ApJ, 688, 789 Giocoli, C., Moreno, J., Sheth, R. K., \& Tormen, G. 2007, MNRAS, 376, 977 González, V., Labbé, I., Bouwens, R. J., et al. 2011, ApJL, 735, L34 Jing, Y. P., Mo, H. J., \& Boerner, G. 1998, ApJ, 494, 1

Komatsu, E., Dunkley, J., Nolta, M. R., et al. 2009, MNRAS, 180, 330 Lacey, C., \& Cole, S. 1993, MNRAS, 262, 627

Lee, K. S., Giavalisco, M., Conroy, C., et al. 2009, ApJ, 695, 368 Madau, P., Pozzetti, L., \& Dickinson, M. 1998, ApJ, 498, 106 Meurer, G. R., Heckman, T. M., \& Calzetti, D. 1999, ApJ, 521, 64 Mo, H. J., \& Fukugita, M. 1996, ApJL, 467, L9 
Oesch, P. A., Bouwens, R. J., Carollo, C. M., et al. 2010, ApJL, 725, L150 Oesch, P. A., Bouwens, R. J., Illingworth, G. D., et al. 2012, ApJ, 745, 110 Peacock, J. A., \& Smith, R. E. 2000, MNRAS, 318, 1144

Reddy, N. A., \& Steidel, C. C. 2009, ApJ, 692, 778

Salpeter, E. E. 1955, ApJ, 121, 161

Sheth, R. K., Mo, H. J., \& Tormen, G. 2001, MNRAS, 323, 1

Sheth, R. K., \& Tormen, G. 1999, MNRAS, 308, 119
Smit, R., Bouwens, R. J., Franx, M., et al. 2012, ApJ, 756, 14

Stark, D. P., Ellis, R. S., Bunker, A., et al. 2009, ApJ, 697, 1493

Stark, D. P., Schenker, M. A., Ellis, R. S., et al. 2013, ApJ, 763, 129

Trenti, M., Stiavelli, M., Bouwens, R. J., et al. 2010, ApJL, 714, L202 Vale, A., \& Ostriker, J. P. 2004, MNRAS, 353, 189

van den Bosch, F. C., Yang, X., \& Mo, H. J. 2003, MNRAS, 340, 771

Zheng, W., Postman, M., Zitrin, A., et al. 2012, Natur, 489, 406 


\section{ERRATUM: “A PHYSICAL MODEL FOR THE $0 \lesssim z \lesssim 8$ REDSHIFT EVOLUTION OF THE GALAXY ULTRAVIOLET LUMINOSITY AND STELLAR MASS FUNCTIONS” (2013, ApJL, 768, L37)

\author{
Sandro Tacchella $^{1}$, Michele Trenti ${ }^{2}$, and C. Marcella Carollo ${ }^{1}$ \\ ${ }^{1}$ Department of Physics, Institute for Astronomy, ETH Zurich, CH-8093 Zurich, Switzerland; tasandro@phys.ethz.ch \\ ${ }^{2}$ School of Physics, University of Melbourne, Parkville, Victoria, Australia \\ Received 2016 May 4; accepted 2016 May 6; published 2016 May 20
}

The normalization of the star formation efficiency reported in Figure 3 (bottom left panel) of the published article is missing a constant factor arising from the conversion from Vega to AB magnitudes. The filter ST-UV14 from the Bruzual \& Charlot (2003) models we used in our model has a magnitude zeropoint offset of $\mathrm{ZP}_{\mathrm{AB}}-\mathrm{ZP}_{\mathrm{VEGA}}=2.5941$, leading to a normalization offset by a factor 10.9051. Figure 3 shows the correct values for star formation efficiencies in the updated bottom left panel. The other results of the Letter remain unaffected by this change.
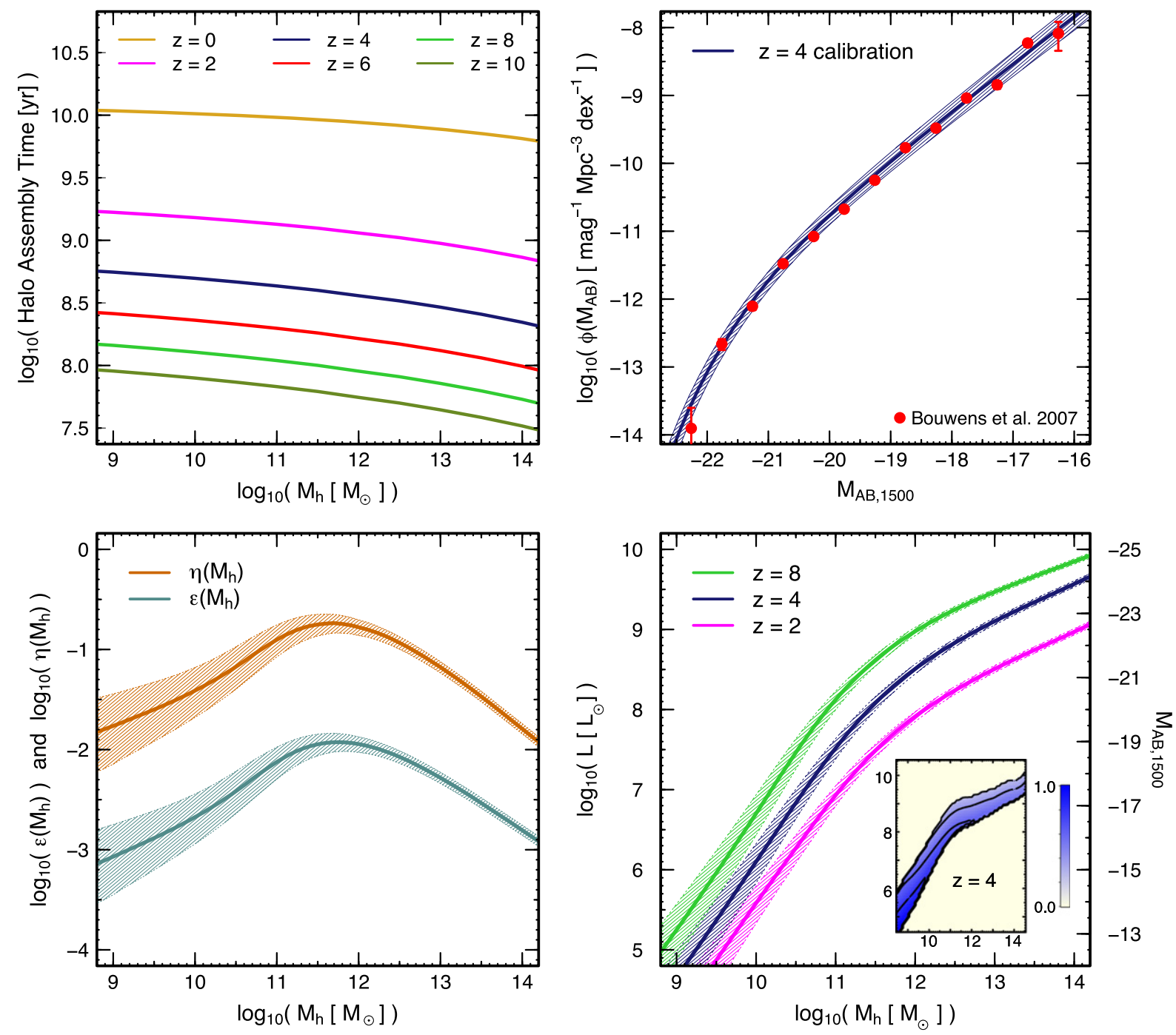

Figure 3. Upper left panel: DM halo assembly time $t_{\text {age }}$ vs. halo mass and redshift. Upper right panel: calibration of our model using the Bouwens et al. (2007) LF at $z=4$. Bottom left panel: star formation efficiencies $\eta\left(M_{h}\right)$ and $\varepsilon\left(M_{h}\right)$ for the burst and accretion mode, respectively. The highest efficiencies are in the range $M_{h} \sim$ $10^{11}-10^{12} \mathrm{M}_{\odot}$. Bottom right panel: the relation between galaxy luminosity and DM halo mass, $L\left(M_{h}, z\right)$, plotted at $z=2,4$, and 8 . In all panels, shaded areas represent $68 \%$ confidence regions. The inset shows the scatter in the $L\left(M_{h}, z\right)$ relation at $z=4$ produced by sampling the whole probability distribution of halo assembly times.

\section{REFERENCES}

Bruzual, G., \& Charlot, S. 2003, MNRAS, 344, 1000

Bouwens, R. J., Illingworth, G. D., Franx, M., \& Ford, H. 2007, ApJ, 670, 928 